\title{
Organizational Form, Information Collection, and The Value of The Firm
}

\author{
Eitan Goldman * \\ Finance Department \\ The Kenan Flagler Business School \\ University of North Carolina \\ Campus Box 3490 McColl Building \\ Chapel Hill, NC 27599-3490 \\ Email: goldmane@bschool.unc.edu \\ Tel: 919-962-9689
}

September 24, 2002

\begin{abstract}
We analyze the impact of organizational form on the incentive of market participants to collect value relevant information about divisions of the firm. We explore whether the market collects less information about divisions of a multi division firm relative to the case in which each division trades as a separate firm. We find that organizational form has a non trivial impact on information collection. In particular, we find that a spinoff may lead to either an increase or a decrease in aggregate information collection. We explore how this result affects firm value and find the conditions under which a spinoff increases and decreases firm value. Our results provide a novel rationale for why firms may choose to spinoff a division or issue a tracking stock.
\end{abstract}

\footnotetext{
${ }^{*}$ I thank Greg Brown, Henry Cao, Jennifer Conrad, Ron Kaniel, Steve Slezak and seminar participants at the 2002 Western Finance Association meetings in Utah, UNC-Chapel Hill, and UT-Austin for their helpful comments and suggestions. All errors remain my own.
} 


\section{Introduction}

Economists have long been interested in understanding the impact of organizational form on internal resource allocation. ${ }^{1}$ This relates to the broader and more fundamental question of determining the optimal size and scope of the firm by identifying the costs and benefits associated with having economic transactions (in this case the allocation of resources) done within the boundary of the firm. In this paper we wish to extend the analysis of the impact of organizational form on resource allocation. However, unlike previous work we are interested in shifting the focus from internal resource allocation to external resource allocation. Namely, we wish to explore the impact of organizational form on the decision of market participants to allocate their resources for the purpose of collecting information about the investments of divisions within the firm and find out how these decisions affect firm value.

The general belief in the market can best be summarized with the following quote from an article, taken from the 2001 September 14 issue of the Wall Street Journal, which talked about the 3Com-Palm spinoff, "Analyst Paul Sgawa... said 3Com is worth more as two pieces than as one. 'I think it's unlocking value for shareholders', he said." In other words the view among practitioners is that spinoffs increase the amount of information in the market and hence increases firm value by bringing stock prices closer to their intrinsic value. Our goal in this paper is to take a rigorous look at this issue.

In practice we see that firms issue tracking stocks (see for example AT\&T), engage in mergers, and spin off divisions. These actions are, at least in part, motivated by the firm's wish to make its core business more easily understood by the market. Our paper can then shed some new light on the possible costs and benefits of these activities.

\footnotetext{
${ }^{1}$ See for example Harris and Raviv(1996), Stein(1997), and Scharfstein and Stein(2000).
} 
The questions which we address in the paper may best be understood with the example of 3Com corporation's recent decision to spin off its Palm Pilot division. This division is now trading as a separate firm called Palm. We ask whether the decision of (potentially) informed traders in the market to collect information about Palm are different now when Palm is trading as a separate company relative to their decision earlier when Palm was a division of $3 \mathrm{Com} .{ }^{2}$ Furthermore, we ask whether the decision to collect information about Palm when it was a division of 3Com was related to the economic characteristics of 3Com's other divisions or whether it was independent of them. In other words if Palm was a division of IBM would the amount of information produced by the market regarding Palm's future prospects have been different? Finally, we ask what are the conditions under which a spinoff will increase the value of the firm?

Understanding the markets incentive to collect information is important for several reasons. In our paper we focus on the role of information in getting stock market prices to move closer to their true (future) value. However, there are other reasons (not explicitly studied in this paper) why informative stock prices may affect firm value. First, stock prices that are informative about future value can help the allocation of resources in the economy. ${ }^{3}$ Second, since stock prices are used in compensation contracts to motivate managers, less informative stock prices will provide very low powered incentives which can potentially lead to bad investment decisions and a reduced or discounted firm value. ${ }^{4}$

\footnotetext{
${ }^{2}$ Note that our focus is on the long run incentive to collect information. In the short term, following a spin off, there are several trading restrictions that may prevent informed investors from trading on their information.

${ }^{3}$ Dow and Gorton(1997) show how informative stock prices can be used by managers to make better investment decisions. Subrahmanyam and Titman(1999) relate the efficiency of stock prices to the development of the capital market.

${ }^{4}$ See Holmstrom and Tirole(1993) for a recent analysis of the monitoring role of stock based compensation.
} 
To address these issues we compare a situation in which the market consists of one multi (two) division firm to a situation in which each of the firm's divisions trade as single division firms. In general, we find that organizational form has a non trivial impact on the amount of information produced/collected by the market and hence on how informative is the stock price regarding the future value of the firm. Perhaps surprisingly, however, we find that following a spinoff (or the creation of a tracking stock) the amount of information produced in the market does not always rise. Rather, holding the total amount of liquidity trades fixed across the two scenarios, we find that spinoffs result in an increase in information collection about one part of the spun off firm and a decrease in information collection about the other part. In sum, a spinoff results in an aggregate increase in information collection only when the divisions have investment sets that have relatively similar Sharpe ratios. Put differently, we find that the likelihood that a spinoff increases aggregate information collection in the market is strictly less than one. This likelihood is higher the smaller the difference between the Sharpe ratios of the investment sets of the two divisions.

The above result implies that the conditions under which a spinoff will increase firm value are more complex then previously thought. A spinoff does not result in an increase in information production about all of the firms assets. Hence, we find that it will increase firm value when it leads to an increase in information production about the part of the firm (division) that is under valued and/or a decrease in information production about the part of the firm that is over valued. This result fits well with the recent empirical work of Allen (2001). In his study he finds that when insiders are net sellers of shares during the first few month following a spinoff the firm has subsequently poor market performance whereas when insiders are net buyers the subsequent performance is positive. While Allen focuses on the predictive power of insider trading following a spinoff his evidence is consistent with 
our story that a spinoff can be motivated both by having an under valued asset (division) and by having an over valued one. Other models (see discussion below) which assume that information production increases after a spinoff cannot explain why a spinoff should take place when some divisions are over valued (i.e., they have subsequent negative performance).

The key to understanding our result is the observation that the optimal amount of (costly) information that is collected in the market depends on the benefit to market participants from trading on their information. There are two main reasons why the incentive to collect information is different for those who trade in the stock of a multi division firm and those who trade in the stocks of single division firm. First, the stock price of a single division firm will only reflect information about the investments made by that division whereas a stock price of a multi division firm will typically incorporate information about all divisions that are within the boundary of that firm. Hence, in a multi division firm information about one division is less valuable because that division constitutes a smaller part of the value of the firm's stock price. Namely, when the informed trader learns about the future value of investments of one division, since she trades on this information using a security whose price is also influenced by investments made by other divisions, her trading profits from collecting information about that division go down. The magnitude of the reduced incentive will typically depend on the relative size and riskiness of the division as it relates to the firm's other divisions. Second, in the multi division firm the informed trader, regardless of her information, trades against the "liquidity traders of the whole firm". However, when the two divisions separate the informed trader trades on her "division $i$ information" only against "division $i$ liquidity traders". Thus, information about division $i$ is more valuable in a multi division firm because the informed traders trades have a smaller impact on the stock price. The equilibrium of the model trades off these two competing effects. 
It is important to point out that we derive these results without specifying exogenously an informational advantage or disadvantage to one organizational form over the other. ${ }^{5}$ Rather we show that if a similar technology can be used to learn about a division, regardless of whether it is a unit of a big firm or if it is a separate entity, it is the strategic choice of market participants to produce different amounts of information, depending on the organizational form, which generates all of our results.

Our paper is based on the literature which deals with information aggregation in stock prices. ${ }^{6}$ We take the modeling framework of Kyle(1985) and build on it to analyze the stock price of a multi division firm. Other related papers which allow for endogenous information collection include Subrahmanyam(1991), Chowdhry and Nanda(1991), and Admati and Pfleiderer(1988). Although our paper focuses on a different aspect of information collection Subrahmanyam(1991) and Gorton and Pennachi (1993) have some similarities to us in that they analyze the benefits of trading a basket of securities. Unlike our paper however they allow for simultaneous trading in both the index and the individual security and analyze where traders will go. In contrast in this paper investors cannot trade the stock of the multi division firm at the same time as they trade in the single division firms since both scenarios are mutually exclusive. In this sense our results relate more closely to a corporate change in the boundary or structure of the firm.

One can view our analysis of spinning off a division as a question of security design. While there is a vast and growing literature on security design ${ }^{7}$ our paper is closest to

\footnotetext{
${ }^{5}$ Habib, Johnson and Naik(1997) assumes that a spinoff results in more informative prices. We on the other hand allow for information collection to be an endogenous choice variable.

${ }^{6}$ For the fundamental papers on the aggregation of information in stock prices see Grossman(1976), Grossman and Stiglitz(1980), Hellwig(1980), and Kyle(1985).

${ }^{7}$ See for example Allen and Gale(1988, 1991), DeMarzo and Duffie (1999)
} 
security design which is motivated by informational issues. ${ }^{8}$ In that regard our paper is similar to that of Boot and Thakor (1993). They study how the mix of securities issued by the firm affect the amount of information collected by the market. They focus on two securities, one that is informationaly sensitive, a stock, and one that is informationaly insensitive, a bond. We on the other hand inherit our security design structure from the corporate structure of the firm (namely its divisions). Our focus is on whether having two informationaly sensitive securities, one for each division, is better than having one security for both.

Finally, our paper also relates to the literature on spinoffs. Aron(1991) demonstrates how a spinoff can improve the incentive contracts written with divisional managers. Habib et al.(1997) show that if a spinoff increases information in stock prices it may lead to better investment decisions by managers. Unlike these two papers we show that, when information collection is endogenous, a purely informational story is sufficient to explain when firms would want to spinoff divisions and when they would not. Namely, we show that even when a spinoff does not affect the compensation package of divisional managers or their investment decisions a spinoff can still increase firm value. Thus, our paper generates fairly distinct empirical predictions about the optimality of a spinoff. As mentioned above these seem to be consistent with the new findings in Allen(2001).

The rest of the paper is organized as follows: In Section 2 we present the model. In Sections 3 and 4 we analyze the economy with single and multi division firms respectively. In Section 5 we compare the two scenarios and discuss the economic interpretation of the differences between them. Section 6 concludes. All proofs are left to the appendix.

\footnotetext{
${ }^{8}$ See Gorton and Pennacchi (1993), Subrahmanyam (1991), and Boot and Thakor (1993).
} 


\section{Model Economy}

We start off with two investment projects which are organized as two separate divisions. The two divisions can either be organized under the roof of one multi division firm or alternatively as two separate single division firms. All firms are assumed to be financed through public equity traded in the open market. Each division receives, at $t=0$, an initial capital investment $\alpha_{i}$ for $i=1,2$. These investments produce, at $t=1$, profits of $\alpha_{i} V_{i}$ for division $i . V_{i}$ denotes the realized return on the project. We make the following assumption about the stochastic properties of returns,

Assumption $1 V_{i}$, is distributed $V_{i} \sim N\left(\mu_{i}, \sigma_{i}^{2}\right)$ for $i=1,2$ and $\operatorname{Cov}\left(V_{1}, V_{2}\right)=0$.

At the initial date, $t=0$, the market does not know what the future return of each project will be. However, there exist an informed trader who can collect information about these projects. Namely, she has a costly technology which allows her to learn about the future profitability of each project. In particular she receives two signals, one on each project, where the amount of information contained in each signal depends on the amount of resources that she puts into getting it. After receiving these signals the informed trader submits a market order for the relevant traded stock(s).

In what follows we describe the model in more detail assuming the two divisions operate as two separate firms. ${ }^{9}$

\section{$2.1 \quad$ Stock Market}

The stock market consists of an informed trader, a continuum of uninformed liquidity traders, and a risk neutral market maker.

\footnotetext{
${ }^{9}$ We postpone the description of the case of a multi division firm to Section 4 . In that section we will discuss how the modeling features change to allow for a fair comparison to the base case above.
} 
The informed trader submits an order for $X_{i}$ shares of firm $i$ based on her information. In contrast the liquidity traders cannot produce information and are subject to a random liquidity shock. In aggregate they submit a random order of $U_{i}$ for shares of Firm $i$ where

$U_{i} \sim N\left(0, \sigma_{u_{i}}^{2}\right)$ and $U_{i}$ is independent of all other random variables. The market maker observes the aggregate demand for each firm, $Y_{i}=X_{i}+U_{i}$, and selects a price, $P_{i}\left(Y_{i}\right)$. We assume that the market maker operates in a competitive market so that he sets prices equal to the expected value of future profits conditional on all available market information. The resulting price then satisfies,

$$
P_{i}\left(Y_{i}\right)=E\left(\alpha_{i} V_{i} \mid Y_{i}, \Omega\right)
$$

Here $\Omega$ denotes the market makers information set at $t=0$ excluding the information contained in the net order flow, $Y_{i}$. In particular $\Omega$ includes information about all model parameters except for the future return, $V_{i}$, and the private signals of the informed trader, $S_{i}$. The modeling of the stock market is closely related to that of Kyle(1985).

\subsection{Informed Trader}

The informed trader is assumed to be risk neutral. Her information set at $t=0$ includes the firms' initial investment decision, $\alpha_{1}$ and $\alpha_{2}$ as well as the parameters of the distributions of the payoffs of each division $\mu_{1}, \sigma_{1}^{2}$ and $\mu_{2}, \sigma_{2}^{2}$. However, she does not know the actual future returns of the divisions, $V_{1}$ and $V_{2}$. For this reason she engages in costly information production. We assume that the informed trader has to expend resources in order to receive a signal about the return of each of the two divisions. Specifically, we assume that,

Assumption 2 The informed trader invests $0 \leq e_{i} \leq 1$ and receives a signal $S_{i}=V_{i}-\mu_{i}+\epsilon_{i}\left(\frac{1-e_{i}}{e_{i}}\right)^{0.5}$ where $\epsilon_{i} \sim N\left(0, \sigma_{i}^{2}\right)$ and $E\left(\epsilon_{i} V_{i}\right)=0$ for $i=1,2$. 
The interpretation of the assumption is that the informed trader receives a separate signal for each project where the signal $S_{i}$ has a zero mean and a variance of $\sigma_{s_{i}}^{2}=\frac{\sigma_{i}^{2}}{e_{i}}$. Thus, producing information about division $i$ increases the accuracy of the signal by decreasing its variance. In the extreme case if the informed trader does not put any resources into collecting the signal $S_{i}$ then the signal she gets is completely uninformative (i.e., $\sigma_{s_{i}}=\infty$ ). If on the other hand she puts in the maximum amount of resources into learning about division $i\left(e_{i}=1\right)$ the signal becomes fully informative in the sense that $S_{i}=V_{i}-\mu_{i}$. In general the more resources put into learning about division $i$, the more informative is the signal she receives. ${ }^{10}$

We further assume that obtaining information is costly,

Assumption 3 The cost of producing information about Firm $i$ is $\frac{1}{2} c_{i} e_{i}^{2}$.

The informed trader collects information on each firms division and then submits a market order to purchase $X_{1}\left(S_{1}\right)$, and $X_{2}\left(S_{2}\right)$ shares of Firm 1 and Firm 2 respectively. These market orders are chosen strategically so as to maximize her expected trading profits, $\pi\left(S_{1}, S_{2}\right)$.

To summarize the model Table 1 provides the sequence of events. ${ }^{11}$

\footnotetext{
${ }^{10}$ Note that $\operatorname{Var}\left(V_{i} \mid S_{i}\right)=\sigma_{i}^{2}\left(1-e_{i}\right)$. Hence, as will be seen in Lemma 3 and Lemma 6 the benefit of collecting information will increase with $\sigma_{i}$. This is because the market makers uncertainty about the future value of each project is $\sigma_{i}$.

${ }^{11}$ All of the main results of the paper can be derived in an analogous model in which there are multiple informed traders who compete with each other. In that model each trader can spend a fixed amount, $e_{i}$, on getting a signal, $S_{i}$, about either Division 1 or Division 2. In equilibrium we solve for the number of traders who choose to become informed about Division 1 and about Division 2. The derivation of the equilibrium in this setting is available upon request.
} 


\section{$3 \quad$ Single Division Firms}

In this section we solve for the information collected by the market when the two divisions trade as two separate firms. We will use the results here as a benchmark to which we can compare the case in which the two divisions merge into one multi division firm.

\subsection{Equilibrium}

The following is the definition of the equilibrium,

Definition 1 The Perfect Bayesian Nash Equilibrium of the game played by the market maker and the informed trader consists of,

(i)An optimal amount of information produced by the informed trader, and an optimal demand for the stocks of each firm based on the information she gets.

(ii) A stock price for each firm which is a function of the market maker's information set and which provides him with an expected profit of zero.

We solve the equilibrium using backward induction. In the first step we solve for the stock prices and the demand functions of the informed trader conditional on her information production decision. In the second step we solve for the informed trader's optimal amount of information production. ${ }^{12}$

The Lemma below provides the stock prices and the optimal demand function of the informed trader when we condition on the amount of information being produced in the market.

\footnotetext{
${ }^{12}$ The equilibrium derived here is similar to an equilibrium where the market maker and the informed trader simultaneously choose the price function and the amount of information to collect, respectively, as a function of what the other player does and then finding the Nash equilibrium $P_{i}$ and $e_{i}$.
} 
Lemma 1 The equilibrium price function is given by $P_{i}=\alpha_{i} \mu_{i}+\lambda_{i} Y_{i}$

where $Y=X_{i}\left(S_{i}\right)+U_{i}$ is the aggregate demand for the firm's stock and where $\lambda_{i}$ is given by,

$$
\lambda_{i}=\frac{1}{2 \sigma_{u_{i}}} \alpha_{i} \frac{\sigma_{i}^{2}}{\sigma_{s_{i}}}
$$

The optimal demand of the informed trader is,

$$
X_{i}\left(S_{i}\right)=\frac{1}{2 \lambda_{i}} \alpha_{i} \frac{\sigma_{i}^{2}}{\sigma_{s_{i}}^{2}} S_{i}
$$

Proof: See Appendix.

Lemma 1 is a simple extension of Kyle(1985) for the case in which the informed trader receives a noisy signal about future returns. Here, $\lambda_{i}$ is a function of $\sigma_{s_{i}}$ and hence will be affected by the endogenous choice of information production (i.e., a function of the endogenous decision variable $e_{i}$ ).

Using Lemma 1 we can proceed to solve for the informed trader's optimal information production. However, we first need to derive the informed trader's expected revenue from her trading activity. This is provided by the lemma below.

Lemma 2 The informed trader's expected revenue from trading on her information, denoted by $\pi\left(e_{1}, e_{2}\right)$, is given by,

$$
\pi\left(e_{1}, e_{2}\right)=\frac{\sigma_{u_{1}}}{2} \frac{\alpha_{1} \sigma_{1}^{2}}{\sigma_{s_{1}}}+\frac{\sigma_{u_{2}}}{2} \frac{\alpha_{2} \sigma_{2}^{2}}{\sigma_{s_{2}}}
$$

where $\sigma_{s_{i}}^{2}=\frac{\sigma_{i}^{2}}{e_{i}}$ for $i=1,2$.

Proof: See Appendix.

The expected revenue is the sum of the revenue generated from trading in Firm 1's stock based on the information collected on that firm's project and the revenue generated from 
trading in Firm 2's stock based on the information collected on Firm 2's project. Note that the expected profit is decreasing in the variance of the signals and therefore is increasing in the amount of information collected, $e_{1}$ and $e_{2}$.

To complete the calculation of the equilibrium we solve below the optimization problem of the informed trader,

$$
\max _{e_{1}, e_{2}} \pi\left(e_{1}, e_{2}\right)-\frac{1}{2} c_{1} e_{1}^{2}-\frac{1}{2} c_{2} e_{2}^{2}
$$

Lemma 3 The optimal amount of information the informed trader collects about the single division firm, Firm i, is,

$$
e_{i}^{S}=\left[\frac{\sigma_{u_{i}}^{2}}{16 c_{i}^{2}} \alpha_{i}^{2} \sigma_{i}^{2}\right]^{\frac{1}{3}}
$$

The amount of information produced about Firm $i$ 's project increases with the amount of capital allocated (internally) to it, $\alpha_{i}$, and with the project return volatility, $\sigma_{i}^{2}$. It also decreases with the parameter for the cost of producing information, $c_{i}$. Not surprisingly it is only a function of the economic parameters of the firm's investment and does not depend on anything that the other firm does.

\section{Multi Division Firm}

In this section we study a multi division firm that has the same investment set as the two single division firms from the previous section. The question we wish to address is whether the new organizational form leads to a change in the amount of information produced in the market. ${ }^{13}$ In order to make a fair comparison of the two economies we need

\footnotetext{
${ }^{13}$ It is important to note that we do not attempt to provide here a complete theory of mergers and spinoff. Admittedly, our goal is merely to provide one additional piece of the big puzzle. We wish to highlight one aspect of organizational form which is the incentive it creates for outsiders to produce information about
} 
to assume that the amount of liquidity in the market is constant across the two scenarios. Specifically, we assume that,

Assumption 4 The variance of liquidity traders in the multi division firm, $\sigma_{u}^{2}$ is equal to $\sigma_{u_{1}}^{2}+\sigma_{u_{2}}^{2}$. Alternatively, we let $\sigma_{u_{i}}^{2}=z_{i} \sigma_{U}^{2}$ where $z_{1}+z_{2}=1$. One can interpret $z_{1}$ as a random variable distributed between zero and one. ${ }^{14}$

We will later refer to $z_{1}$ as $z$ and $z_{2}$ as $1-z$.

In addition to the above assumption we have,

Assumption 5 The informed trader gets the same signals, $S_{1}$ and $S_{2}$ as before.

The only difference between the two cases then is in that the informed trader now has one equity security which she can use to make her trades. Unlike the previous case where she was able to trade on her information in two securities, one for each single division firm, here there is one security which aggregates all the information about both divisions.

The Lemma below describes the equilibrium price and demand of the informed trader.

Lemma 4 The equilibrium price function is given by $P=\mu+\lambda Y$ where $Y=X\left(S_{1}, S_{2}\right)+U$ is the aggregate demand for the firm's stock and where $\mu$ and $\lambda$ are given by, $\mu=\alpha_{1} \mu_{1}+\alpha_{2} \mu_{2}$ and

$$
\lambda=\frac{1}{2 \sigma_{u}}\left[\alpha_{1}^{2} \frac{\sigma_{1}^{4}}{\sigma_{s_{1}}^{2}}+\alpha_{2}^{2} \frac{\sigma_{2}^{4}}{\sigma_{s_{2}}^{2}}\right]^{0.5} .
$$

internal projects. In that sense we do not capture many of the important elements that may be associated with multi division firms such as better/worse internal resource allocation, better/worse coordination and greater/smaller agency problems. We hope, however, that restricting our attention to the current issues may still provide valuable insight.

${ }^{14}$ We follow the literature by assuming that liquidity traders are non strategic. As will be shown below our results hold for any value of $z_{i}$. 
The optimal demand of the informed trader is,

$$
X\left(S_{1}, S_{2}\right)=\frac{1}{2 \lambda}\left[\alpha_{1} \frac{\sigma_{1}^{2}}{\sigma_{s_{1}}^{2}} S_{1}+\alpha_{2} \frac{\sigma_{2}^{2}}{\sigma_{s_{2}}^{2}} S_{2}\right]
$$

Proof: See Appendix.

From Lemma 4 we can see the impact of having two divisions inside one firm. Since there is only one traded security the market maker selects a price function which aggregates all available information regarding both divisions into one price. For this reason the market depth parameter $\lambda$ is now a function of $\alpha_{1}$ and $\sigma_{s_{1}}$ as well as of $\alpha_{2}$ and $\sigma_{s_{2}}$. In addition, the demand function of the informed trader is linear and additive in both signals $S_{1}$ and $S_{2}$. For a given signal $S_{j}$ the higher is her signal $S_{i}$ the higher will be her demand for the stock.

Lemma 5 The informed trader's expected revenue from trading on her information, denoted by $\pi^{M}\left(e_{1}, e_{2}\right)$, is given by,

$$
\pi^{M}\left(e_{1}, e_{2}\right)=\frac{\sigma_{u}}{2}\left[\frac{\alpha_{1}^{2} \sigma_{1}^{4}}{\sigma_{s_{1}}^{2}}+\frac{\alpha_{2}^{2} \sigma_{2}^{4}}{\sigma_{s_{2}}^{2}}\right]^{0.5}
$$

Proof: See Appendix.

As before, the expected profits are decreasing in the variance of the signal and therefore increasing in the amount of information produced. More interesting perhaps is the impact of collecting information about one division on the informed traders total expected trading profits. Unlike the single division setting, here the profit function is no longer additively separable in the trading profits generated from each signal. From equation 8 we see that trading on information about Division 1 results in profits associated with the term $\frac{\alpha_{1}^{2} \sigma_{1}^{4}}{\sigma_{s_{1}}^{2}}$ while trading on information about Division 2 results in profits associated with the term $\frac{\alpha_{2}^{2} \sigma_{2}^{4}}{\sigma_{s_{2}}^{2}}$. Due to the fact that both divisions are traded under one security these two terms are no longer additively separable. 
To solve for the informed trader's information production decision we maximize

$$
\max _{e_{1}, e_{2}} \pi^{M}\left(e_{1}, e_{2}\right)-\frac{1}{2} c_{1} e_{1}^{2}-\frac{1}{2} c_{2} e_{2}^{2}
$$

which can be written as,

$$
\max _{e_{1}, e_{2}} \frac{\sigma_{u}}{2}\left[\alpha_{1}^{2} \sigma_{1}^{2} e_{1}+\alpha_{2}^{2} \sigma_{2}^{2} e_{2}\right]^{0.5}-\frac{1}{2} c_{1} e_{1}^{2}-\frac{1}{2} c_{2} e_{2}^{2}
$$

Lemma 6 The informed trader's optimal allocation of resources when producing information about divisions 1 and 2 of the multi division firm is,

$$
\begin{aligned}
& e_{1}^{M}=\left[\frac{\sigma_{u}^{2}}{16} \frac{c_{2}}{c_{1}^{2}} \frac{A^{3}}{A^{2} c_{2}+B^{2} c_{1}}\right]^{\frac{1}{3}} \\
& e_{2}^{M}=\left[\frac{\sigma_{u}^{2}}{16} \frac{c_{1}}{c_{2}^{2}} \frac{B^{3}}{A^{2} c_{2}+B^{2} c_{1}}\right]^{\frac{1}{3}}
\end{aligned}
$$

where $A=\alpha_{1}^{2} \sigma_{1}^{2}$ and $B=\alpha_{2}^{2} \sigma_{2}^{2}$.

Proof: See Appendix.

Lemma 6 completes the equilibrium. Combined with Lemma 3 this lemma is the key to understanding the main results of the paper. In the Corollary below we explore the implications of Lemma 6.

Corollary 1 The equilibrium amount of information collected about division $i, e_{i}^{M}$, is increasing in the size of capital investment in division $i, \alpha_{i}$, and the volatility of its profits, $\sigma_{i}^{2}$

Intuitively, if the division gets more funds it will represents a larger part of the total firm and therefore will have a bigger impact on the stock price. In that case it becomes more critical for whoever wants to trade in the stock to learn about the operation of that division. The reason why the informed trader also collects more information if the division invests 
in projects with a higher volatility of future revenue is because higher volatility results in higher uncertainty for the whole market (i.e., the market maker and the uninformed trader). Since the informed trader is competing against the market maker the value of becoming informed about something which the market is more uncertain about will generate higher average trading profits. This means that there is a greater benefit to the informed trader from reducing her uncertainty by increasing the accuracy of the signal she gets. ${ }^{15}$

Corollary 2 The amount of information production regarding division $i$ depends on the economic characteristic of other divisions within the boundary of the firm, namely division $j \neq i$. Specifically, information acquisition $e_{i}^{M}$ decreases with $\alpha_{j}$ and $\sigma_{j}^{2}$.

Corollary 2 demonstrates one aspect of the impact of organizational form on the decision to collect information about a division of the multi division firm. The difference between the results here and the results in Lemma 3 highlight the impact of the boundary of the firm on the markets incentive to collect information. Here, since both divisions trade under the umbrella of one firm there is one security which aggregates all available information. In practical terms this means that trading on information regarding both Divisions 1 and 2 is done against the liquidity traders of the whole firm (1.e., $\sigma_{u}$ ). Therefore, the incentive to produce information about one division depends on how big it is relative to the other division and on how volatile its profits are relative to the other division. In particular, if $\alpha_{j}$ goes up the relative impact of the outcome of division $i$ 's project on the performance of the stock goes down. This in turn reduces the incentive to collect information about division $i$. Hence $e_{i}^{M}$ goes down. Similarly, if $\sigma_{j}$ goes up the volatility of the stock price associated with division $j$ 's profits goes up too. In that case the relative value of gathering information about division $i$ goes down. In essence then the link between information collection about

\footnotetext{
${ }^{15}$ Note that the last result is strongly related to the assumed structure of the signal (Assumption 2).
} 
one division and the other is due to the fact the informed trader faces a joint liquidity constraint when she trades based on the two sources of information she gets.

\section{Single Versus Multi Division Firm}

In this section we analyze the differences between the equilibrium amount of information collected in the two economies, the magnitude of these differences and their economic implications. The variables we use to compare across the two economies are the total amount of information produced in the market, the volatility of prices, and firm value.

To flush out the economic interpretation of the results we will assume for sake of concreteness that the investments, $\alpha_{i}$, are chosen endogenously. Specifically we assume that the manager(s) solves,

$$
\max _{\alpha} \alpha \mu_{i}-\frac{1}{2} \alpha^{2} \sigma_{i}^{2}
$$

so that $\alpha_{i}=\frac{\mu_{i}}{\sigma_{i}^{2}}$ for $i=1,2 .^{16}$

While not critical to the analysis we assume for notational simplicity that $c_{1}=c_{2}$. Also it will be convenient to define $\frac{\mu_{1}}{\sigma_{1}}=\beta G$ and $\frac{\mu_{2}}{\sigma_{2}}=\frac{1}{\beta} G$. Note that $\beta$ captures the level of divergence between the investment opportunity sets of the two divisions. When $\beta=1$ the divisions have investment sets which have the same Sharpe ratios while for $\beta \neq 1$ the investment sets diverge (symmetrically). Finally, we assume with out loss of generality that $\beta>1$. This is equivalent to the assumption that the investment set of Division 1 is better than that of the other division.

Most of the intuition for the comparisons will come from the following relationship which

\footnotetext{
${ }^{16}$ While it is true that divisional investment may change depending on whether the division is part of a multi division firm or not we ignore this issue in the current paper. Instead we focus on changes that are purely due to changes in market information production.
} 
is derived from Lemma 2 and Lemma 6.

$$
e_{i}^{M}=e_{i}^{S}\left[\frac{1}{z_{i}} \frac{\left(\frac{\mu_{i}}{\sigma_{i}}\right)^{4}}{\left(\frac{\mu_{i}}{\sigma_{i}}\right)^{4}+\left(\frac{\mu_{j}}{\sigma_{j}}\right)^{4}}\right]^{\frac{1}{3}}
$$

Equation 11 shows the relationship between the amount of information collected about a division when it is part of a multi division firm and the amount of information collected about the same division when it trades as a separate firm.

As a first observation note that since $\frac{\left(\frac{\mu_{i}}{\sigma_{i}}\right)^{4}}{\left(\frac{\mu_{i}}{\sigma_{i}}\right)^{4}+\left(\frac{\mu_{j}}{\sigma_{j}}\right)^{4}}+\frac{\left(\frac{\mu_{j}}{\sigma_{j}}\right)^{4}}{\left(\frac{\mu_{i}}{\sigma_{i}}\right)^{4}+\left(\frac{\mu_{j}}{\sigma_{j}}\right)^{4}}=1$ and since $z_{1}+z_{2}=1$ we have that for any $z_{i}$,

$$
\begin{aligned}
e_{i}^{M}=e_{i}^{S} \text { and } e_{j}^{M}=e_{j}^{S} & \text { if and only if } & z_{i}=\frac{\left(\frac{\mu_{i}}{\sigma_{i}}\right)^{4}}{\left(\frac{\mu_{i}}{\sigma_{i}}\right)^{4}+\left(\frac{\mu_{j}}{\sigma_{j}}\right)^{4}} \\
e_{i}^{M}>e_{i}^{S} \text { and } e_{j}^{M}<e_{j}^{S} & \text { if and only if } & z_{i}<\frac{\left(\frac{\mu_{i}}{\sigma_{i}}\right)^{4}}{\left(\frac{\mu_{i}}{\sigma_{i}}\right)^{4}+\left(\frac{\mu_{j}}{\sigma_{j}}\right)^{4}} \\
e_{i}^{M}<e_{i}^{S} \text { and } e_{j}^{M}>e_{j}^{S} & \text { if and only if } & z_{i}>\frac{\left(\frac{\mu_{i}}{\sigma_{i}}\right)^{4}}{\left(\frac{\mu_{i}}{\sigma_{i}}\right)^{4}+\left(\frac{\mu_{j}}{\sigma_{j}}\right)^{4}}
\end{aligned}
$$

This means that following a spinoff (or prior to a merger) organizational form does matter for information collection. Typically, a spinoff results in an increase in the amount of information produced about one division and a decrease in the amount of information produced about the other division. For a given level of liquidity distribution, $z$, which of the two divisions will see an increase in information collection will depend on how different the investment sets of the two divisions are (i.e., how different are their Sharpe ratios).

The question left open is what is the impact of the change of organizational form on aggregate levels of information production, volatility of stock prices, and firm value. This is the focus of the propositions below. 
Proposition 1 For any liquidity distribution $z$ there exists a level of diversity $\beta^{*}(z)$ such that for $\beta>\beta^{*}(z)$ a spinoff will decrease the aggregate amount of information production and for $\beta<\beta^{*}(z)$ a spinoff will increase information production. For $\beta=\beta^{*}(z)$ information production does not change following a spinoff.

\section{Proof of Proposition 1}

The proof follows immediately once one realizes that, $e_{1}^{S}+e_{2}^{S}=\left(\frac{\sigma_{u}^{2}}{16}\right)^{\frac{1}{3}}\left[z_{1}^{\frac{1}{3}} A^{\frac{1}{3}}+z_{2}^{\frac{1}{3}} B^{\frac{1}{3}}\right]$, and $e_{1}^{M}+e_{2}^{M}=\left(\frac{\sigma_{u}^{2}}{16}\right)^{\frac{1}{3}}\left[\left(\frac{A^{2}}{A^{2}+B^{2}}\right)^{\frac{1}{3}} A^{\frac{1}{3}}+\left(\frac{B^{2}}{A^{2}+B^{2}}\right)^{\frac{1}{3}} B^{\frac{1}{3}}\right]$. Replacing $A$ with $(\beta G)^{2}$ and $B$ with $\left(\frac{1}{\beta} G\right)^{2}$ we get that spinoffs lead to more information if and only if

$$
z^{\frac{1}{3}} \beta^{\frac{4}{3}}+(1-z)^{\frac{1}{3}} 1 \geq\left(\frac{\beta^{4}}{\beta^{4}+\beta^{-4}}\right)^{\frac{1}{3}} \beta^{\frac{4}{3}}+\left(\frac{\beta^{-4}}{\beta^{4}+\beta^{-4}}\right)^{\frac{1}{3}} 1
$$

Hence, a spinoff increases information if and only if $\beta<\beta^{*}(z)$ where $\beta^{*}(z)$ solves $z=\frac{\beta^{* 4}}{\beta^{* 4}+\beta^{*-4}}$.

Proposition 1 shows that a spinoff does not always increase the aggregate amount of information being produced in the market. Rather, we see that for any distribution of liquidity, $z$, spinoffs will result in more information production if and only if the two divisions have investment sets whose Sharpe ratios are not too different.

The intuition is based on the observation that the value from trading on information increases with both the liquidity level and with the Sharpe measure of each division (see Lemma's 3 and 6). With two firms the amount of information collected about each firm is (roughly speaking) proportional to its Sharpe measure and to its stock liquidity. Since the total amount of liquidity is fixed the more liquidity going to the firm with the high Sharpe measure the higher will be the aggregate amount of information produced in the market.

With a multi division firm information about each division is also proportional to its divisional Sharpe measure. But here it is proportional to the liquidity of the whole firm. 
This is the benefit of collecting information about a multi division firm. However, due to the confounding impact of each division on the profit from collecting information about the other, the information collected on each division decreases by the ratio of the divisions Sharpe measure to the sum of the Sharpe measures of all divisions. Thus, the more diversity in Sharpe measures the higher is the aggregate amount of information collected in this case. If then the divisions are sufficiently diverse the benefit of having greater liquidity will dominate the negative impact of having two sources of information in one stock price.

The intuition above is at the basis of all the analysis to follow. Hence, it is worth repeating the key differences in the incentive to collect information between the two scenarios. In the multi division firm the incentive to collect information is decreased because in order to gain from having information about one division you have to trade in a stock that also reacts to the returns from the other division. However, the incentive to collect information is increased because the liquidity of the stock is higher. The opposite holds for the single division firm. Here, there is no confounding information from the other division but there is less liquidity to trade against.

To be concrete, if a small IT company was to merge with Microsoft the increased liquidity that this firm will get when being part of Microsoft may outweigh the fact that it is now a small part of a big corporation. Hence, the merger may result in more information production in the market.

If one considers the liquidity trades, $z$, as being a random variable then the result above has a natural empirical implication: One is more likely to see a spinoff that results in an increase in information production whenever the difference between the investment sets of the two divisions is smaller.

Proposition 1 demonstrates that the general perception that spinoffs always increase 
information is not correct. We show that both a merger and a spinoff can result in an increase in information. The equilibria in which multi division firms can have a stock price that contains more information about future value might explain why many firms choose not to spinoff divisions. Also, these equilibria offer a rationale for why firms choose not to issue tracking stocks which, on the face of it, seem to be a good idea. The equilibria of our model demonstrate that issuing a tracking stock does not always increase information collection. In particular, if the tracking stock attracts too much of the liquidity away from the main firm, or if the Sharpe ratios of investments are sufficiently different, the issuance can result in a loss of information. ${ }^{17}$

Below we analyze what happens to the unconditional volatility of the stock price.

Corollary 3 The volatility of the stock price, $P$, and $P_{i}$, of the multi and single division firm is given by,

$$
\begin{gathered}
\operatorname{Var}(P)=\frac{1}{2}\left(\alpha_{1}^{2} \sigma_{1}^{2} e_{1}^{M}+\alpha_{2}^{2} \sigma_{2}^{2} e_{2}^{M}\right) \quad \text { and } \\
\operatorname{Var}\left(P_{i}\right)=\frac{1}{2} \alpha_{i}^{2} \sigma_{i}^{2} e_{i}^{S}
\end{gathered}
$$

respectively. Hence, the stock market is more volatile following a spinoff if and only if the condition in Proposition 1 above holds.

\subsection{Firm Value}

In this section we explore the conditions under which a spinoff will result in a higher firm value. To analyze the value of the firm we assume that after making the investment

\footnotetext{
${ }^{17}$ Note that in the context of our model spinoffs are in essence equivalent to issuing a tracking stock for one of the two divisions. This is because our spinoff does not involve any change in the divisions production function. Thus, one can also view our paper in the context of the literature on security design.
} 
decision the manager observes $V_{1}$ and $V_{2}$ and based on this she decides whether to spinoff a division. In addition, we assume that

Assumption 6 The informed trader makes her trading decision only based on her noisy signals, $S_{i}$ and not based on the spinoff decision of the manager.

This assumption simplifies the complexity of the equilibrium by allowing us to ignore the potential signaling effect of the organizational choice. While this assumption may be problematic we believe that it does not change the main intuitions provided below. ${ }^{18}$

We can now turn to calculate the expected value of the firm from the managers perspective. When both divisions trade as a multi division firm the expected price of the firm, conditional on $V_{1}$ and $V_{2}$ is

$$
E\left(P(Y) \mid V_{1}, V_{2}\right)=\alpha_{1} \mu_{1}+\alpha_{2} \mu_{2}+\frac{1}{2} \alpha_{1} e_{1}^{M}\left(V_{1}-\mu_{1}\right)+\frac{1}{2} \alpha_{2} e_{2}^{M}\left(V_{2}-\mu_{2}\right)
$$

The expected price is calculated based on the equilibrium price function given in Lemma 4. When the two divisions trade as two separate firms the sum of their expected values is,

$$
E\left(P_{1}\left(Y_{1}\right) \mid V_{1}\right)+E\left(P_{2}\left(Y_{2}\right) \mid V_{2}\right)=\alpha_{1} \mu_{1}+\alpha_{2} \mu_{2}+\frac{1}{2} \alpha_{1} e_{1}^{s}\left(V_{1}-\mu_{1}\right)+\frac{1}{2} \alpha_{2} e_{2}^{s}\left(V_{2}-\mu_{2}\right)
$$

This calculation is based on the equilibrium values in Lemma 1. Looking at equations 14 and 15 it is clear that when information production is assumed to be a constant exogenous number the expected values under both scenarios are the same. Therefore the difference in value between the spinoff and the non-spinoff cases will be, again, due to endogenous information production.

\footnotetext{
${ }^{18}$ In a somewhat similar setting Boot and Thakor(1993) solve for a pooling equilibrium by assuming that the value of the firm has a bimodal distribution. Since we have two divisions whose values are normally distributed the signaling model that they look at becomes intractable here.
} 
Proposition 2 For any distribution of liquidity trades $z$ there exists a constant $\beta^{*}(z)$ such that for $\beta>\beta^{*}(z)$ a sufficient condition for spinoffs to be non-profitable is that, $V_{1}>\mu_{1}$ and that $V_{2}<\mu_{2}$. Further a sufficient condition for spinoffs to be profitable is that, $V_{1}<\mu_{1}$ and that $V_{2}>\mu_{2}$. The opposite holds for $\beta<\beta^{*}(z)$.

Proof: See Appendix

A spinoff may or may not increase the value of the firm. If the Sharpe ratios of the two divisions are sufficiently diverse a spinoff increases value when the (future) return of the division with the higher Sharpe measure is below its expected return and the (future) return of the other division is above its expected return. The reason being that for $\beta>\beta^{*}(z)$ a spinoff will result in a decrease in information production about Division 1 and an increase in information production about Division 2. Less information about Division 1 is valuable when the true return $V_{1}$ is lower than the expected return $\mu_{1}$ and more information is valuable when $V_{2}$ is greater than $\mu_{2}$.

The intriguing aspect of a spinoff is that it does not result in an increase in information production about all divisions. Therefore, a spinoff will increase firm value when it leads to an increase in information production about the undervalued part of the firm and/or when it leads to a decrease in information production about the over valued part of the firm. This implies that spinoffs can be motivated by both having undervalued assets and having over valued ones. As mentioned earlier this can explain the recent empirical findings in Allen(2001) which show that a spinoff can also occur when insiders have information that a division is over valued. 


\subsection{Liquidity Traders}

Although our results are true for any level of $z$ one element which is left un modeled in the analysis is what determines where liquidity traders go when there are two traded stocks. In other words what are the characteristics of the firm which attract liquidity traders? The problem is that liquidity traders are inherently non strategic agents. Other studies which have modeled strategic liquidity traders either assume that liquidity traders are subject to exogenous endowment shocks and hence they trade for hedging reasons (see for example Spiegel and Subrahmanyam (1992)), or they assume that these traders are divided into groups of discretionary and non discretionary liquidity traders (see for example Admati and Pfleiderer(1988) and Subrahmanyam (1991)). Unfortunately, in our case both of these modeling improvements do not provide any additional insight. If we assume that liquidity traders trade for hedging reasons then clearly they would be better off trading in the two single division firms. Thus, we would probably have that $\sigma_{u}^{2}<\sigma_{u_{1}}^{2}+\sigma_{u_{2}}^{2}$. However, our equilibrium is determined by both (1) the difference $\sigma_{u}^{2}-\left(\sigma_{u_{1}}^{2}+\sigma_{u_{2}}^{2}\right)$ and (2) how $\sigma_{u_{1}}$ relates to $\sigma_{u_{2}}$. The problem then is that both (1) and (2) will be a function of the utility of the liquidity traders and of their endowment shocks. Since both of these must be given exogenously it is not clear that there is a benefit to adding this layer of complexity. If we choose to go the route of dividing the liquidity traders into discretionary and non discretionary traders then we would still have that $\sigma_{u}^{2}=\sigma_{u_{1}}^{2}+\sigma_{u_{2}}^{2}$ so the equilibrium will be determined by the relation between $\sigma_{u_{1}}$ and $\sigma_{u_{2}}$. This relation however will be determined by the exogenous assumption regarding what firm characteristics attract the non discretionary traders and what is the fraction of traders that are non discretionary. Thus, again it is not clear whether this benefits our analysis.

For the above reasons we leave the liquidity traders as non strategic agents with the 
belief that the main results of our paper that: 1) organizational form matters for information production and that 2)spinoffs can increase firm value due to either having an under valued division or to having an over valued one, are robust to a more detailed description of these agents.

\section{Conclusion}

In this paper we analyze the impact of organizational form on the incentive of market participants to collect information about the future value of investments made by the firm. We show that the incentive to collect information about a division depends on whether that division is traded as a separate firm or whether it is traded as part of a multi division firm. We show that in a multi division setting information collection regarding a specific division is affected not only by the economic characteristics of that division but also by the relative size and volatility of that division as they relate to the rest of the firm. We demonstrate the implications of these results for the aggregate amount of information in the market and for the value of the firm. Our analysis suggests that spinoffs do not always increase the amount of information produced in the stock market and do not always increase firm value. Whether a spinoff leads to an increase in information production and in firm value depends on how similar are the Sharpe ratios of the investment sets of the two divisions. 


\section{Appendix}

\section{Proof of Lemma 1 and 4}

The proof is for Lemma 4. For a proof of Lemma 1 simply set $\alpha_{i}=0$ and $\sigma_{u}=\sigma_{u_{i}}$ to get $\lambda_{j}, X_{j}$ and $P_{j}$. We look for a linear equilibrium where, given the two signals $S_{1}$ and $S_{2}$, the market maker sets a price $P=\mu+\lambda Y$ where $Y=X+U$ is the aggregate demand. The two conditions that must be satisfied in equilibrium are:

$$
\begin{aligned}
& \text { (i) } \quad P=E\{V \mid X+U\} \\
& \text { (ii) } X=\operatorname{argmax}_{x} E\left\{x(V-P) \mid S_{1}, S_{2}\right\} \text {. }
\end{aligned}
$$

Here, $V=\alpha_{1} V_{1}+\alpha_{2} V_{2}$.

$(i)$ is the condition that the market maker selects a price which gives him zero expected profits and $(i i)$ is the condition that the informed trader submits a demand which maximizes her trading profits.

(i) and $(i i)$ can be written as,

$$
\begin{gathered}
P=\alpha_{1}\left\{\mu_{1}+\frac{\operatorname{Cov}\left(V_{1}, Y\right)}{\sigma_{Y}^{2}}\left(Y-\mu_{Y}\right)\right\}+\alpha_{2}\left\{\mu_{2}+\frac{\operatorname{Cov}\left(V_{2}, Y\right)}{\sigma_{Y}^{2}}\left(Y-\mu_{Y}\right)\right\} \\
X=\frac{1}{2 \lambda}\left[\alpha_{1} E\left(V_{1} \mid S_{1}\right)+\alpha_{2} E\left(V_{2} \mid S_{2}\right)-\mu\right]
\end{gathered}
$$

respectively. If we denote the informed trader's demand as $X=a+b_{1} S_{1}+b_{2} S_{2}$ then we have the following four equations:

$$
\begin{array}{r}
X=a+b_{1} S_{1}+b_{2} S_{2} \\
X=\frac{1}{2 \lambda}\left[\alpha_{1}\left(\mu_{1}+\frac{\sigma_{1}^{2}}{\sigma_{s_{1}}^{2}} S_{1}\right)+\alpha_{2}\left(\mu_{2}+\frac{\sigma_{2}^{2}}{\sigma_{s_{2}}^{2}} S_{2}\right)-\mu\right] \\
P=\mu+\lambda Y \\
P=\alpha_{1} \mu_{1}+\alpha_{2} \mu_{2}-a\left(\alpha_{1} \frac{b_{1} \sigma_{1}^{2}}{\sigma_{Y}^{2}}+\alpha_{2} \frac{b_{2} \sigma_{2}^{2}}{\sigma_{Y}^{2}}\right)+Y\left(\alpha_{1} \frac{b_{1} \sigma_{1}^{2}}{\sigma_{Y}^{2}}+\alpha_{2} \frac{b_{2} \sigma_{2}^{2}}{\sigma_{Y}^{2}}\right)
\end{array}
$$


Comparing coefficients we get five conditions that need be satisfied in equilibrium:

$$
\begin{array}{r}
a=\frac{1}{2 \lambda}\left(\alpha_{1} \mu_{1}+\alpha_{2} \mu_{2}-\mu\right) \\
b_{1}=\frac{\alpha_{1} \sigma_{1}^{2}}{2 \lambda \sigma_{s_{1}}^{2}} \\
b_{2}=\frac{\alpha_{2} \sigma_{2}^{2}}{2 \lambda \sigma_{s_{2}}^{2}} \\
\mu=\alpha_{1} \mu_{1}+\alpha_{2} \mu_{2}-a \lambda \\
\lambda=\frac{1}{\sigma_{Y}^{2}}\left(\alpha_{1} b_{1} \sigma_{1}^{2}+\alpha_{2} b_{2} \sigma_{2}^{2}\right)
\end{array}
$$

The solution to this set of equations is

$$
\begin{gathered}
a=0, \text { and } \mu=\alpha_{1} \mu_{1}+\alpha_{2} \mu_{2}, \\
\lambda=\frac{1}{2 \sigma_{u}}\left[\alpha_{1}^{2} \frac{\sigma_{1}^{4}}{\sigma_{s_{1}}^{2}}+\alpha_{2}^{2} \frac{\sigma_{2}^{4}}{\sigma_{s_{2}}^{2}}\right]^{0.5}, \\
b_{1}=\alpha_{1} \sigma_{u} \frac{\sigma_{s_{2}}}{\sigma_{s_{1}}} \sigma_{1}^{2}\left[\frac{1}{\alpha_{1}^{2} \sigma_{1}^{4} \sigma_{s_{2}}^{2}+\alpha_{2}^{2} \sigma_{2}^{4} \sigma_{s_{1}}^{2}}\right]^{0.5}, \\
b_{2}=\alpha_{2} \sigma_{u} \frac{\sigma_{s_{1}}}{\sigma_{s_{2}}} \sigma_{2}^{2}\left[\frac{1}{\alpha_{1}^{2} \sigma_{1}^{4} \sigma_{s_{2}}^{2}+\alpha_{2}^{2} \sigma_{2}^{4} \sigma_{s_{1}}^{2}}\right]^{0.5} .
\end{gathered}
$$

The final calculation of $X\left(S_{1}, S_{2}\right)$ can be derived directly from the above equations and the proof follows.

\section{Proof of Lemma 2 and Lemma 5}

This is a proof of Lemma 5. To get the proof for Lemma 2 simply note that, $\pi\left(e_{1}, e_{2}\right)=$ $\pi_{1}\left(e_{1}\right)+\pi_{2}\left(e_{2}\right)$ where $\pi_{i}\left(e_{i}\right)=\pi^{M}\left(e_{i}, e_{j}=0\right)$ and where we replace $\sigma_{u}$ with $\sigma_{u_{i}}$ where appropriate.

The informed traders expected revenue from trading prior to making the investment decision in information production is,

$$
\pi^{M}\left(e_{1}, e_{2}\right)=E\left(E\left[X(V-P(Y)) \mid S_{1}, S_{2}\right]\right)=\operatorname{Cov}(X, V)-\lambda \operatorname{Var}(X)
$$


Now, $\operatorname{Cov}(X, V)=\alpha_{1} b_{1} \sigma_{1}^{2}+\alpha_{2} b_{2} \sigma_{2}^{2}$ and $\operatorname{Var}(X)=b_{1}^{2} \sigma_{s_{1}}^{2}+b_{2}^{2} \sigma_{s_{2}}^{2}$. Using the expressions derived in Lemma 1 for $\lambda, b_{1}$ and $b_{2}$ the lemma follows.

Proof of Lemma 6 Taking the first order condition with respect to $e_{1}$ and $e_{2}$ we have,

$$
\begin{aligned}
& \frac{\sigma_{u}}{4} \alpha_{1}^{2} \sigma_{1}^{2}\left[\alpha_{1}^{2} \sigma_{1}^{2} e_{1}+\alpha_{2}^{2} \sigma_{2}^{2} e_{2}\right]^{-0.5}-c_{1} e_{1}=0 \\
& \frac{\sigma_{u}}{4} \alpha_{2}^{2} \sigma_{2}^{2}\left[\alpha_{1}^{2} \sigma_{1}^{2} e_{1}+\alpha_{2}^{2} \sigma_{2}^{2} e_{2}\right]^{-0.5}-c_{2} e_{2}=0
\end{aligned}
$$

Taking the first equation and dividing it by the second we find that $e_{1}=\frac{\alpha_{1}^{2} \sigma_{1}^{2} c_{2}}{\alpha_{2}^{2} \sigma_{2}^{2} c_{1}} e_{2}$. We now can replace this expression for $e_{1}$ in one of the first order conditions to get that $\frac{4 c_{2} e_{2}}{B \sigma_{U}}\left[\frac{A^{2} c_{2}}{B c_{1}} e_{2}+B e_{2}\right]^{0.5}=1$. The last equation can then be simplified to obtain $e_{2}^{M}$. A similar analysis will provide us with a solution to $e_{1}^{M}$ and the proof follows.

Proof of Proposition 2 By definition the expected profit from a spinoff is equal to the difference between equations 15 and 14 .

$$
D=\frac{1}{2} \alpha_{1}\left(e_{1}^{s}-e_{1}^{M}\right)\left(V_{1}-\mu_{1}\right)+\frac{1}{2} \alpha_{2}\left(e_{1}^{s}-e_{2}^{M}\right)\left(V_{2}-\mu_{2}\right)
$$

Using equation 11 we have,

$$
e_{1}^{s}-e_{1}^{M}=e_{1}^{s}\left[1-\left(\frac{1}{z} \frac{(\beta G)^{4}}{(\beta G)^{4}+\left(\frac{1}{\beta} G\right)^{4}}\right)^{\frac{1}{3}}\right]
$$

and,

$$
e_{2}^{s}-e_{2}^{M}=e_{2}^{s}\left[1-\left(\frac{1}{1-z} \frac{\left(\frac{1}{\beta} G\right)^{4}}{(\beta G)^{4}+\left(\frac{1}{\beta} G\right)^{4}}\right)^{\frac{1}{3}}\right]
$$

Thus, we can rewrite $D$ as,

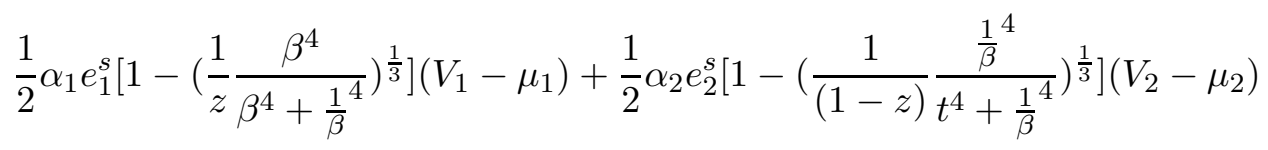

After some more algebra we get that $D>0$ if and only if,

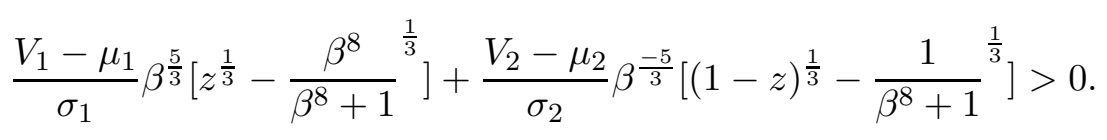


Now, define $\beta^{*}(z)$ by the $\beta$ for which $\frac{\beta^{4}}{\beta^{4}+\beta^{-4}}=z$. Then for $\beta>\beta^{*}(z)$ the term in the first square brackets is negative and the term in the second square brackets is positive. Thus, the proof follows. 


\section{References}

[1] Admati A., and P. Pfleiderer, 1988, "A Theory of Interday Patterns: Volume and Price Variability," Review of Financial Studies, 1, 3-40.

[2] Allen, F., and Gale, D., 1988, "Optimal Security Design," Review of Financial Studies, 1, 229-264.

[3] Allen, F., and Gale, D., "Arbitrage, Short Sales, and Financial Innovation," Econometrica, 59, 1041-1068.

[4] Allen, J., 2001, "Private Information and Spin-off Performance," Journal of Business, $74(2), 281-306$.

[5] Aron, D., 1991, "Using the Capital Market as a Monitor: Corporate Spin-offs in an Agency Framework," Rand Journal of Economics, 22, 505-518.

[6] Boot, A., and Thakor, A., 1993, "Security Design," Journal of Finance, 48, 1349-1378.

[7] Chowdhry B., and V. Nanda, 1991, "Multimarket Trading and Market Liquidity," Review of Financial Studies, 4(3), 483-511.

[8] DeMarzo P., and D. Duffie, 1999, “A Liquidity-Based Model of Security Design,” Econometrica, 67, 65-99.

[9] Dow J., and G. Gorton, 1997, "Stock Market Efficiency and Economic Efficiency: Is There a Connection?" Journal of Finance, 52, 1087-1129.

[10] Grossman S., 1976, "On the Efficiency of Competitive Stock Markets Where Traders have Diverse Information," Journal of Finance, 31, 573-585. 
[11] Grossman S., and J. Stiglitz, 1980, "On The Impossibility of Informationaly Efficient Markets "American Economic Review, 70, 393-408.

[12] Gorton G., and G. Pennacchi, 1993, "Security Baskets and Index-linked Securities" Journal of Business, 66, 1-27.

[13] Habib M., A. B. Johnson, and N. Naik, 1997, "Spinoffs and Information," Journal of Financial Intermediation, 6, 153-176.

[14] Harris M., and A. Raviv, 1996, "The Capital Budgeting Process, Incentives and Information," Journal of Finance, 51, 1139-1174.

[15] Hellwig M., 1980, "On the Aggregation of Information in Competitive Markets," Journal of Economic Theory, 22, 477-498.

[16] Holmstrom B., and J. Tirole, 1993, "Market Liquidity and Performance Monitoring," Journal of Political Economy, 101, 678-709.

[17] Kyle A., 1985, "Continuous Auctions and Insider Trading," Econometrica, 53, 13151335.

[18] Scharfstein D., and J. Stein, 2000, “The Dark Side of Internal Capital Markets: Divisional Rent-Seeking and Inefficient Investment," Journal of Finance, 55, 2537-64.

[19] Stein J., 1997, "Internal Capital Markets and the Competition for Corporate Resources," Journal of Finance, 52, 111-133.

[20] Spiegel M., and A., Subrahmanyam, 1992, "Informed Speculation and Hedging in a Noncompetitive Securities Market," Review of Financial Studies, 5(2), 307-29. 
[21] Subrahmanyam A., 1991, "A Theory of Trading in Stock Index Futures," Review of Financial Studies, 4(1), 17-51.

[22] Subrahmanyam A., and S. Titman, 1999, "The Going-Public Decision and The Development of Financial Markets," Journal of Finance, 54, 1045-1082. 
TABLE 1

\begin{tabular}{lll}
$t=0$ & & $t=1$ \\
\hline Informed trader & Market maker & $V_{1}$ and $V_{2}$ \\
invests $e_{1}, e_{2}$, & selects $P_{i}\left(Y_{i}\right)$ & are realized, \\
gets signals & firms are liquidated, and \\
$S_{1}\left(e_{1}\right), S_{2}\left(e_{2}\right)$ shareholders consume profits & \\
Informed trader submits demand & & \\
$X_{1}\left(S_{1}\right), X_{2}\left(S_{2}\right)$, & \\
and uninformed submit demand $U_{i}$. &
\end{tabular}

